Bond University

Research Repository

\title{
Bonverstr
}

\section{Artifact: The Feasibility of Dense Indoor LoRaWAN towards Passively Sensing Human Presence}

Grubel, Jascha; Thrash, Tyler; Helal, Didier; Sumner, Robert W.; Holscher, Christoph; Schinazi, Victor R.

Published in:

2021 IEEE International Conference on Pervasive Computing and Communications Workshops and other Affiliated Events (PerCom Workshops)

DOI:

10.1109/PerComWorkshops51409.2021.9431070

Licence:

Other

Link to output in Bond University research repository.

Recommended citation(APA):

Grubel, J., Thrash, T., Helal, D., Sumner, R. W., Holscher, C., \& Schinazi, V. R. (2021). Artifact: The Feasibility of Dense Indoor LoRaWAN towards Passively Sensing Human Presence. In 2021 IEEE International Conference on Pervasive Computing and Communications Workshops and other Affiliated Events (PerCom Workshops) (pp. 444-445). [9431070] (2021 IEEE International Conference on Pervasive Computing and Communications Workshops and other Affiliated Events, PerCom Workshops 2021). IEEE, Institute of Electrical and Electronics Engineers. https://doi.org/10.1109/PerComWorkshops51409.2021.9431070

\section{General rights}

Copyright and moral rights for the publications made accessible in the public portal are retained by the authors and/or other copyright owners and it is a condition of accessing publications that users recognise and abide by the legal requirements associated with these rights.

For more information, or if you believe that this document breaches copyright, please contact the Bond University research repository coordinator. 


\title{
Artifact: The Feasibility of Dense Indoor LoRaWAN Towards Passively Sensing Human Presence
}

\author{
Jascha Grübel $^{*}$, Tyler Thrash ${ }^{\dagger}$, Didier Hélal ${ }^{\ddagger}$, Robert W. Sumner*, Christoph Hölscher*, Victor R. Schinazi ${ }^{\S}$ \\ * ETH Zürich, Zürich, Switzerland \\ Email: jascha.gruebel@inf.ethz.ch, robert.sumner@inf.ethz.ch, christoph.hoelscher@gess.ethz.ch \\ $\dagger$ Miami University, Oxford, OH, USA \\ Email: thrashst@miamioh.edu \\ $\ddagger$ Orbiwise, Geneva, Switzerland \\ Email: didier.helal@orbiwise.com \\ $\S$ Bond University, Robina, Australia \\ Email: victor_schinazi@bond.edu.au
}

\section{INTRODUCTION}

We present a large data set of 14 million entries of indoor Long Range Wide Area Network (LoRaWAN) transmission metadata to study Dense Indoor Sensor Networks (DISN) [1]. We also provide a mixed model implementation in an R-Markdown file for analyzing the transmissions based on the metadata to obtain all transmission-related results of the main paper. The data set will regularly expand as the system continues to collect data until at least 2022.

Understanding Long Range Wide Area Network (LoRaWAN) transmissions indoors is critical to test the feasibility of Dense Indoor Sensor Networks (DISN). While LoRaWAN has been widely studied outdoors, we believe that indoor setups require a different conceptualization to account for signal shadowing and multi-path fading. The data set has been used in the accompanying paper to compute an indoor model of LoRaWAN transmissions. We provide the transmission data to enable other researchers to develop alternative indoor models for comparisons. The data set consists of all the LoRaWAN transmissions from the first six months of the prototype of the digital twin described in the paper. The data has been exported from the PostgreSQL data base on the EVEREST server described in Section III-B of the manuscript. The annotated transmission data was supplied by the LoRaWAN network server via webhook and stored on our server. The data has been stripped of identifying information with respect to the building because of privacy concerns.

\section{DATA DESCRIPTION}

The public data set consists of a single table containing 21 characteristics of 14 million received transmissions (see Table I). Not included in the public data set are geometric relations between sensors and gateways because of privacy concerns.

In the data set, each row represents the reception of transmissions on the network server and is uniquely identified by the combination of the identifying variables transmission, device, and gateway. Under LoRaWAN, a transmission of a device can be received by any gateway, and therefore, a single transmission may appear up to 3 times, once per gateway. In some scenarios, there may be less transmission due to environmental (e.g., wall material) or network factors (e.g., gateway up-time).

The devices remained in the same location during the 6 months of data collection and have several immutable characteristics. The device_type corresponds to the 5 sensor types presented in Table II of the section III-A of the paper. The distance between gateway and device was computed with the PostGIS extension. The device locations were predetermined during installation along with a building information model (BIM). Similarly, the gateway floor gw_floor and device floor dev_floor were annotated during installation, and the floor distance floor_dist was directly computed from the difference between the two. We only used floor_dist for modeling, but the other two variables can be used for visualization. The BIM indicates that a floor has a height of $3.36 \mathrm{~m}$ which was used to calculate maximal transmission distances.

Transmission characteristics vary with each transmission and describe the LoRa configuration, network meta data on the signal strength, and varying device properties. The LoRa configuration is described in the Data Rate (DR) dr, the Spreading Factor (SF) sf, the frequency freq, and the Time-on-Air (ToA) toa. The network metadata consists of the measured snr and rssi, the arrival time time, and a Boolean flag main_gw that indicates which was the main recipient gateway with the strongest received signal. The device property that is transmission-specific is the frame_count, indicating the internal ID of the transmission within the device. The frame count allows us to determine the Frame Error Rate (FER). A gap in the frame count indicates that the device has sent a transmission that was not received by the network.

The derived measures determine two types of concurrent transmissions. First, multi-channel concurrent transmissions concurrent occur at the same time but on a different channel and SF and have no impact on the transmission quality. Second, destructive concurrent transmissions 
TABLE I

COLUMN DESCRIPTION FOR THE DATA Frame OF LORAWAN TRANSMISSIONS.

\begin{tabular}{|c|c|c|c|c|}
\hline Variable & Description & Data Type & Unit & Range \\
\hline \multicolumn{5}{|l|}{ Identifying variables } \\
\hline transmission & ID of transmission & Integer & ID & $2-6645488$ \\
\hline device & ID of device & Integer & ID & $1-136 \& 140-393$ \\
\hline \multicolumn{5}{|c|}{ Device characteristics } \\
\hline device_type & main sensor type & Integer & ID & $\begin{array}{r}\text { 1=Orbiwise noise; } 2=\text { ERS PIR; } 3=\text { ERS CO2; } \\
4=\text { Browan PIR; } 5 \text { = Browan VOC }\end{array}$ \\
\hline gw_floor & Floor of gateway & Integer & Descriptive & $0-7$ \\
\hline floor_dist & $\begin{array}{l}\text { Number of floors between } \\
\text { gateway and device }\end{array}$ & Integer & Count & $0-7$ \\
\hline \multicolumn{5}{|l|}{ Transmission characteristics } \\
\hline$d r$ & Data rate used & String & Descriptive & "SF[7-12]BW125" \\
\hline sf & Spreading factor used & Integer & Descriptive & $7-12$ \\
\hline freq & Frequency used & Integer & $\mathrm{Hz}$ & $867100000-868500000$ \\
\hline main_gw & $\begin{array}{l}\text { main gateway that } \\
\text { received the data }\end{array}$ & Boolean & Logical & True-False \\
\hline frame_count & Sensor-based frame counter & Integer & Count & $1-56739$ \\
\hline \multicolumn{5}{|c|}{ Derived measures } \\
\hline interval_start & Equal to time minus toa & String & ISO 8601 Date \& Time & 2020-02-25 14:39:00-2020-09-10 12:33:14 \\
\hline interval_end & Equal to time & String & ISO 8601 Date \& Time & 2020-02-25 14:39:00-2020-09-10 12:33:14 \\
\hline interval_start_buffer & Start interval with $25 \%$ buffer & String & ISO 8601 Date \& Time & 2020-02-25 14:39:00-2020-09-10 12:33:14 \\
\hline interval_end_buffer & End interval with $25 \%$ buffer & String & ISO 8601 Date \& Time & $2020-02-25 \quad 14: 39: 00-2020-09-10 \quad 12: 33: 14$ \\
\hline concurrent & $\begin{array}{l}\text { Other transmissions that do not } \\
\text { impact this transmission }\end{array}$ & Integer & Count & 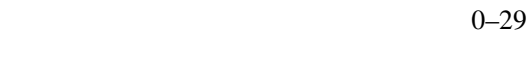 \\
\hline concurrent_destructive & $\begin{array}{l}\text { Other transmissions that could } \\
\text { cancel this transmissions }\end{array}$ & Integer & Count & $0-5$ \\
\hline
\end{tabular}

concurrent_destructive on the same frequency and SF make the reception of all transmissions at a single gateway impossible. However, the physical distance combined with short ToA make it possible that a destructive concurrent transmission at one gateway is non-concurrent at another. We employ multiple gateways to detect destructive concurrent transmissions. First, the transmission interval is based on the time and toa described with the variables interval_start and interval_end. Since our gateways are network synchronized, we must allow for a delay (up to $20 \mathrm{~ms}$ ). We use a conservative estimate of $25 \%$ of toa to buffer the interval on both ends described in interval_start_buffer and interval_end_buffer. The buffered intervals are used to compute concurrent and concurrent_destructive.

\section{Data AVAILABILITY}

The data set is accessible on Zenodo as a CSV file: https://doi.org/10.5281/zenodo.4476317. Zenodo is a service of CERN that allows free upload of scientific data. A single submission (up to 50GB) is never deleted as long as CERN is operating. Currently, this promises data availability at least for the next 20 years with a high probability that the funding agencies behind CERN will extend the operation indefinitely.

We withhold some information because of privacy concerns. For more information, please contact the first author.

\section{Result VAlidation}

In addition, the Zenodo repository contains an R Markdown file to reproduce the model and model predictions in the main paper. At least 16GB RAM are required to run the models.

1) Install and open RStudio (https://rstudio.com/products/ rstudio/download/).

2) Open 2021_PerCom_mixed_model.Rmd.

3) Ensure that LoRaWAN_DISN_transmission_meta -data.csv is in the same folder.

4) Press $C t r l+A l t+R$ to run the code.

All models and graphs from the main paper are reproduced as shown in the knitr output files of the above steps: 2021_PerCom_mixed_model.[pdf/html].

\section{ACKNOWLEDGMENT}

This research is funded by grant ETH-15 16-2 and supported by OrbiWise with hard- and software. We thank Michal GathMorad for the BIM.

\section{REFERENCES}

[1] J. Grübel, T. Thrash, D. Hélal, R. W. Sumner, C. Hölscher, and V. R. Schinazi, "The feasibility of dense indoor lorawan towards passively sensing human presence," in 2021 IEEE International Conference on Pervasive Computing and Communications (PerCom), 2021, pp. 1-2. 